\title{
Forest-Fire Analogy to Explain the $b$ Value of the Gutenberg-Richter Law for Earthquakes
}

\author{
E. A. Jagla \\ Centro Atómico Bariloche and Instituto Balseiro, Comisión Nacional de Energía Atómica, (8400) Bariloche, Argentina
}

(Received 6 May 2013; published 3 December 2013)

\begin{abstract}
The Drössel-Schwabl model of forest fires can be interpreted in a coarse-grained sense as a model for the stress distribution in a single planar fault. Fires in the model are then translated to earthquakes. I show that when a second class of trees that propagate fire only after some finite time is introduced in the model, secondary fires (analogous to aftershocks) are generated, and the statistics of events becomes quantitatively compatible with the Gutenberg-Richter law for earthquakes, with a realistic value of the $b$ exponent. The change in exponent is analytically demonstrated in a simplified percolation scenario. Experimental consequences of the proposed mechanism are indicated.
\end{abstract}

DOI: $10.1103 /$ PhysRevLett.111.238501

PACS numbers: 91.30.Ab, 05.65.+b, 91.30.Px

The size distribution of earthquakes (EQs) follows the empirical Gutenberg-Richter (GR) law [1,2], stating that the number of EQs in a small magnitude interval $d M$ is $\sim 10^{-b M} d M$. This law has been observed to be accurate in a wide range of magnitudes with values of $b$ ranging from 0.8 to 1.2. When expressed in terms of the seismic moment $S$ (such that, up to a constant, $M=\frac{2}{3} \log _{10} S$ ), the number $d N$ of EQs with a seismic moment between $S$ and $S+d S$ is given by $d N=N(S) d S \sim S^{-\tau} d S$ with $\tau=1+\frac{2}{3} b$, so realistic values of $\tau$ are in the range 1.5-1.8.

From the point of view of statistical physics, the GR law has been rationalized in the context of self-organized critical systems [3]. However, this scenario does not explain the value of the $b$ exponent experimentally observed. Statistical models devised to describe EQs [4] (most remarkably, the Burridge-Knopoff [5] and Olami-FederChristensen [6] models) typically display a GR law over a relatively wide magnitude range. However, unless some parameter is tuned, they are not able to reproduce the correct value of the $b$ exponent.

In the past years, models including the possibility of relaxation effects within the plates (related to plastic effects, creep dynamics, etc.) have been presented [7-9]. An interesting characteristic of these models is that they justify the existence of aftershocks (ASs), related to the relaxation effects. Although some of these models reproduce the GR law with the correct $b$ exponent, even in the limited context of the models themselves, it is not obvious why this numerical value appears.

An appropriate scenario to discuss the origin of the $b \simeq 1$ value turns out to be the Drössel-Schwabl (DS) model of forest fires $[10,11]$. Although introduced to model a very different problem, it has been recognized that a coarsegrained version of this model may qualitatively represent the evolution of stress in a single planar fault system [12-14]. I will show that the introduction of a second kind of trees in the model, that propagate fire only after some finite time, generates secondary fires analogous to aftershocks and a realistic value of the $b$ exponent.
I summarize here the rules of the DS model for completeness. In an initially empty, two-dimensional square lattice with periodic boundary conditions, sites are sequentially occupied at random by trees, one every time unit $t_{0}$. After $r$ tree insertions, a lightning event occurs at some random position, burning the tree that is located there, and all trees that can be reached from this site through nearest neighbor occupied sites. This instantaneous burning defines the fires in the system, their size (the number of burnt trees) being denoted by $S$. In the limit in which $r \rightarrow \infty$, there are fires with arbitrarily large values of $S$. There is evidence that the system does not become truly critical in this limit $[15,16]$; however, a size distribution of fires with an exponent around $\tau \sim 1.2$ has been consistently observed for a wide range of sizes, and this is sufficient for the analysis presented here.

A coarse-grained variable in the model is the density of trees over some finite size region. This density can take values from 0 to 1 and can be thought to represent the stress state of the plate. The random addition of trees can be considered, in the coarse-grained sense, as a smooth increase of the stress in the system, caused by external tectonic loading. When stress is high in some portion of the system, it can be abruptly reduced by random lightning to some small value, through the occurrence of a fire that is interpreted in this view as an EQ. The microscopic stochastic laws of the DS model prevent it from reaching a globally synchronized oscillatory state. The wide distribution of fires in the DS model corresponds to a wide distribution of earthquakes. However, the exponent $\tau \sim 1.2$ observed in the DS model does not correspond to the exponent for EQs, around 1.7. Also, as the system lacks an internal dynamics, ASs are not produced. We will see that a single additional ingredient in the model is able to produce ASs and a GR law with a realistic $\tau$ exponent.

I propose a modified DS model by introducing a second species of trees, called $B$ trees (the original ones are denoted $A$ trees). The rules of the modified model are the following. In the insertion step, each time a site is chosen 
for insertion and if this site is empty, it becomes occupied by a $B$ tree with probability $w$ and by an $A$ tree with probability $1-w$. The value of $w$ will be assumed to be small $(w \ll 1)$. In the burning step, $A$ trees burn and propagate fire instantaneously to all neighbors, irrespective of whether they are $A$ or $B$ trees. However, $B$ trees are supposed to propagate fire to neighbors only after some random time of the order of a new time scale $t_{1}$. This time scale $t_{1}$ is supposed to be much shorter than the insertion time $t_{0}$ yet much larger than the propagation of fire through $A$ trees. An example of a time sequence of fires in this modified DS model is shown in Fig. 1. On a given initial configuration of $A$ and $B$ tress, lightning strikes at some random site. This produces an instantaneous fire that burns all trees connected to this site, and that "activates" the $B$ trees highlighted in Fig. 1(b), which retard the propagation for some time of the order of $t_{1}$. As these times for individual $B$ trees are considered to be random, for the implementation I simply choose one of the active $B$ trees randomly and start a secondary fire [Fig. 1(c)]. The process is continued until no tree is burning [Fig. 1(d)]. At this stage, the insertion process is continued.

Time sequences of fires consist now of "clusters" of events that are triggered by lightning and are separated by the time $t_{0}$. Within a cluster, each event is triggered by an active $B$ tree. I will assume for simplicity that the delay
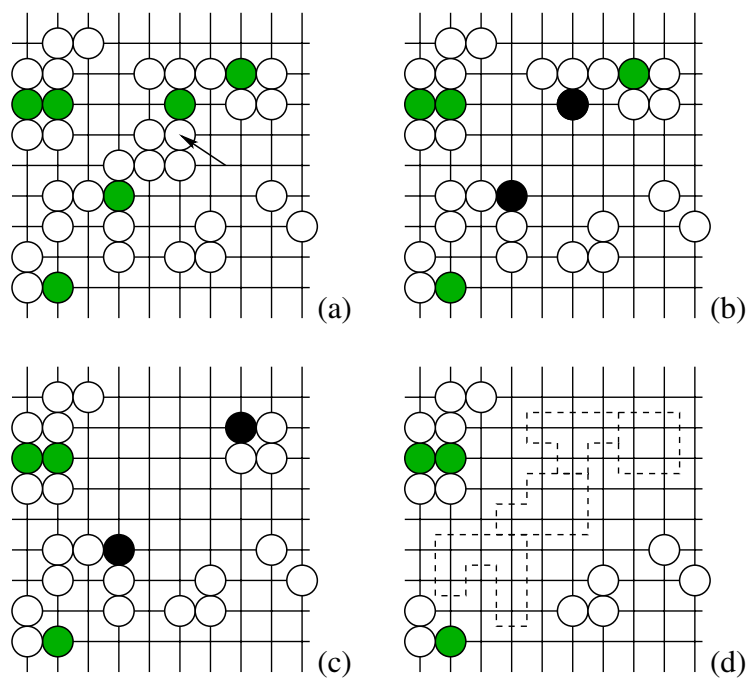

FIG. 1 (color online). Propagation of fires in the modified DS model. Circles indicate sites occupied by $A$ (white) or $B$ [gray (green online)] trees. (a) On some initial configuration, lightning strikes the site indicated by the arrow. (b) The state after the instantaneous burning of the connected set of $A$ trees. The two $B$ trees that are highlighted as black have become "active." (c) One of the active $B$ sites is chosen at random and propagates fire to its neighbor trees. Additional $B$ sites may become active. (d) Final stage when there are no more active $B$ sites. Along the whole process, fires of sizes 5, 4, 4, and 6 have occurred (contours are highlighted). The total size of the burnt cluster is thus 19 . time of each $B$ tree has a Poisson distribution with average value $t_{1}$. In the EQ analogy, secondary fires are the ASs, and active $B$ trees are their epicenters. The typical number of ASs observed depends on the parameter $w$, fixing the ratio between $B$ and $A$ trees at insertion. Clusters of events correspond to the initial shock and all its ASs. The assumed condition $t_{1} / t_{0} \rightarrow 0$ allows one to clearly identify ASs in the model, a situation that is not fulfilled in actual seismicity.

A temporal sequence of events for the modified DS model is presented in Fig. 2. The clustered structure of the sequence is apparent. Secondary fires to given initial lightning occur at the same value of $t / t_{0}$, and thus they appear on the same vertical line. The plot in Fig. 2(b) shows the same events but plotted as a function of the internal time within the cluster $t / t_{1}$, with the value of $t$ set to 0 at the initial lightning of every cluster. The average AS rate displays an exponential time decay with a time constant $t_{1}$. The real AS rate decays in time as a power law (Omori-Utsu [17] law). This discrepancy indicates that the single internal time scale $t_{1} \mathrm{I}$ am assuming is not very
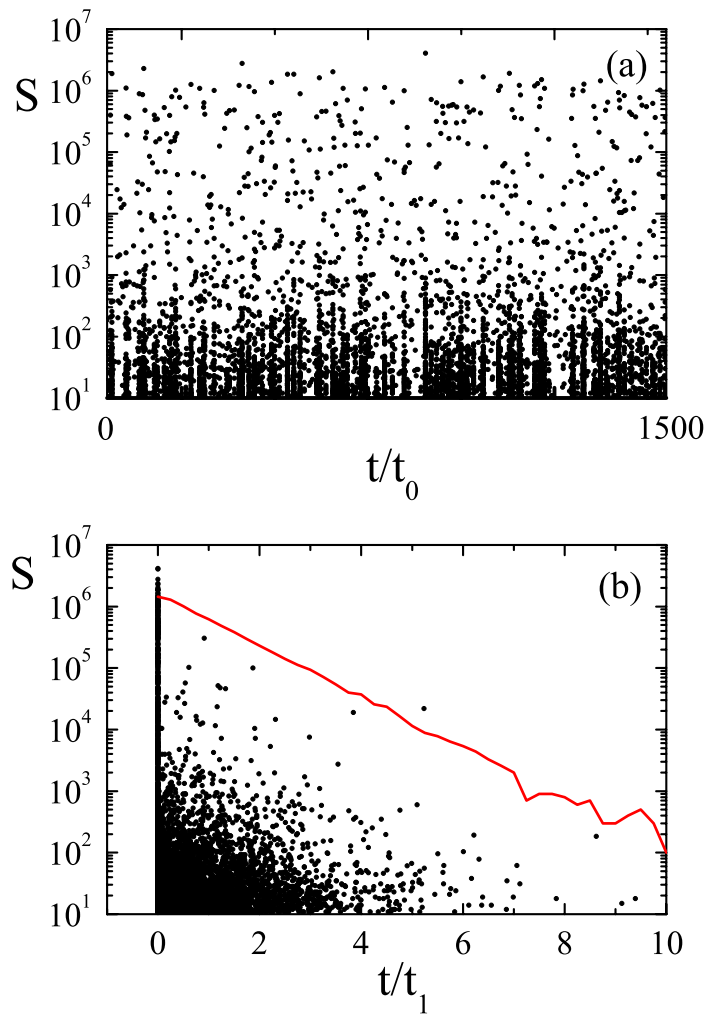

FIG. 2 (color online). (a) Temporal sequence of fires, in the modified DS model on a system of size $4000 \times 4000$, with $r=$ $5 \times 10^{4}$ and $w=10^{-3}$. Events that occur at the same value of $t / t_{0}$ form what I have called a cluster, corresponding to the initial fire, generated by lightning, and all secondary fires propagated through $B$ trees. In (b), the same events are plotted as a function of its internal time within the cluster, set to 0 for the first event of the cluster. The continuous line is the average AS rate. 
realistic in this respect, but this does not alter the main conclusions below.

Size distributions of events are presented in Fig. 3 with open symbols. The presence of $B$ trees generates a size distribution with a $\tau$ exponent in the range 1.7-1.8, well different from the original slope $(\tau \simeq 1.2)$ and comparable to the value of the actual GR law. Systematic results (presented in Supplemental Material [18]) show that $w$ has to be small enough in order to have a wide size range in which the new trend is followed. Although a perfect power law cannot be guaranteed with the present simulations, I note that for $w$ in the range $10^{-3}-10^{-2}$ the new power law is observed in a range of $S$ values that is comparable to the experimental range for EQs. The slope change generated by the presence of $B$ trees (by the existence of ASs, in the seismic perspective) is the main result of this work, and I will now explain its origin.

First of all, I notice that, in addition to calculating the size distribution of individual events, it is also interesting to calculate the size distribution of clusters. Namely, I define the cluster size $S_{C}$ as the sum of sizes of the initial event and all its secondary fires. The size of the cluster $S_{C}$ is exactly what we had obtained as a single event if $B$ trees also propagate fire instantaneously or, equivalently, if there are no $B$ trees at all $(w=0)$. In fact, the distribution of clusters, plotted in Fig. 3 with full symbols, coincides with the distribution in the original model. Thus we see that the effect of $B$ trees is to fragment the clusters in pieces that burn at different times. This fragmentation process, at the same time that it generates secondary fires, produces the change in the exponent of the size distribution.

It is useful to have an unambiguous characterization of this effect in a simpler model than the DS forest fire. In fact, in the DS model the spatial distribution of trees is highly correlated, and rigorous results for the size distribution of avalanches are not available. However, the

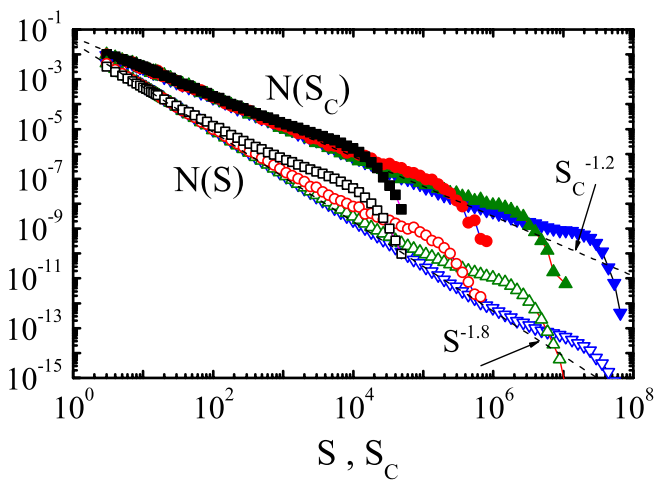

FIG. 3 (color online). Statistics of events for simulations with $r=10^{3}, 10^{4}, 10^{5}$, and $10^{6}$ (from left to right) and $w=0.01$ (system size is up to $20000 \times 20000$ ). Lower curves correspond to individual events, whereas the upper ones are the results for the complete clusters. The statistics of clusters coincides with the result for single events in the case $w=0$. analysis becomes much simpler if we consider the propagation of fires on a totally uncorrelated distribution of $A$ and $B$ trees, with spatial densities $\rho_{A}$ and $\rho_{B}$ (which now have to be fixed by hand). This problem can be studied with the tools of standard percolation theory [19]. If no $B$ trees are present $\left(\rho_{B}=0\right)$, events correspond to those that occur in a site percolation problem with probability $\rho_{A}$. As $\rho_{A} \rightarrow$ $p_{c} \simeq 0.5927$, the events become power law distributed with an exponent $187 / 91-1 \simeq 1.055$ [19]. In the presence of $B$ trees, we can calculate again the distribution of single events and the distribution of clusters. Clusters are distributed as if $B$ trees are not present; i.e., they correspond to normal percolation, with a cutoff fixed by the total density $\rho_{A}+\rho_{B}\left(<p_{c}\right.$, to avoid an infinite fire). For single events instead, we have the same fragmentation effect discussed in the context of the DS model. The clusters become diluted with a fraction $\rho_{B} /\left(\rho_{A}+\rho_{B}\right)$ of $B$ trees, generating a fragmentation effect. The size distribution of single events is the size distribution of these fragments. The effect of fragmenting a percolation cluster by the removal of a small fraction of sites was studied in [20], where it was shown that even the removal of a single site can split the cluster in two. When this happens, and close to the percolation threshold, the fragments are distributed according to a power law with an exponent $\phi$, the fragmentation exponent, that was found to be $\phi \simeq 1.60$ in two dimensions.

Results of numerical simulations in the two-species version of the percolation problem (see results in Supplemental Material [18]) confirm this view: The inclusion of a small fraction of $B$ trees changes the distribution of events to a new power law with a $\tau \simeq 1$.60. A perfect power law is obtained in the limit $\rho_{A}+\rho_{B} \rightarrow p_{c}$, $\rho_{B} /\left[p_{c}-\left(\rho_{A}+\rho_{B}\right)\right] \rightarrow$ const. If $\rho_{B}$ is kept finite as $\rho_{A}+\rho_{B} \rightarrow p_{c}$, a noncritical distribution is obtained, with the maximum size of the fragments controlled by the value of $\rho_{A}$.

The present analysis of the modified DS model and the percolation limit points out a simple mechanism that can justify the observed value of the $b$ exponent of the GR law in the context of a single planar fault situation. It suggests that the occurrence of this value is closely related to the presence of aftershocks. It also points out a few possibilities to observe experimental signatures that would be a consequence of this mechanism. One of them is the fact that the statistics of clusters must display a less steep decay than that of individual EQs. An actual verification of this fact would imply the unambiguous classification of events in clusters, which is a nontrivial task, since the $t_{0}$ and $t_{1}$ time scales are not well separated in actual seismicity. However, there have been promising attempts to group the EQs in clusters according to appropriate metrics in the space-time-magnitude parameter space (see, for instance, $[21,22]$ ), that may serve this purpose. A careful study of experimental data is needed to advance in this direction. 

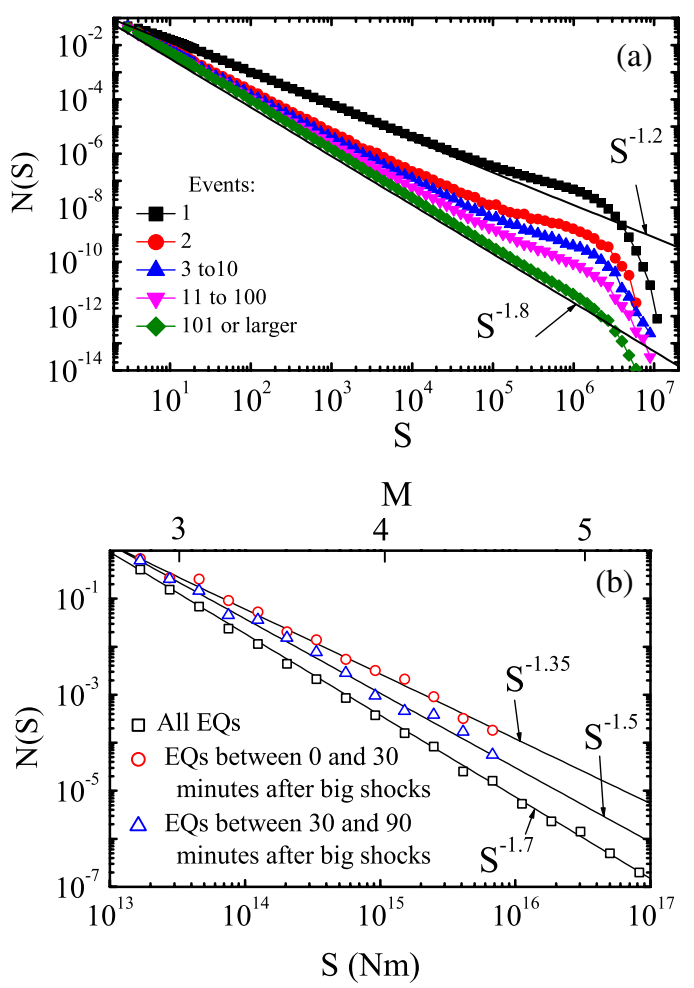

FIG. 4 (color online). (a) Partial size distribution of events in the modified DS model ( $\left.r=10^{5}, w=0.01\right)$, restricted to the indicated events in the clusters. The distribution has an exponent close to that of the original model for the initial event and becomes progressively steeper as successive secondary events are considered. (b) An actual example of this phenomenon (details are provided in Supplemental Material [18]). The lower points display the distribution of EQs in Southern California during a 20-year time period. The upper curves correspond to the distributions of events that occurred in the two time windows indicated, after EQs of magnitude 4.5 or larger. Note that these two partial distribution are limited to magnitudes lower than 4.5, since larger events are counted as main shocks. The behavior is qualitatively similar to that in (a). Straight lines are for reference only.

A second signature indicating that the proposed mechanism may be relevant in actual seismicity is the following. If we classify events by an index $m$ indicating their temporal location in the AS sequence (with $m=1$ for the initial shock) and calculate the size distributions in small windows of $m$, the result is that shown in Fig. 4(a). It is seen that the decaying of the distribution has an exponent similar to that of the whole clusters for the initial shock and becomes progressively steeper as successive events within the sequence are considered. This behavior can be understood by considering again the simpler percolation scenario. In that case, the very first event in each cluster is taken from a distribution with a density $\rho_{A}$ of occupied sites, and this has to follow a decaying law with the smaller $\tau(\simeq 1.055$ in the percolation case). Successive ASs progressively drive the distribution towards the new, larger $\tau$ value.
This effect is suitable for experimental verification. In fact, EQ catalogs are dominated, after large magnitude events, by the ASs to those events. Although other events that are not ASs certainly occur, they are few in the first hours after large events. So they make a minor contribution that can be neglected in a first approach. In Fig. 4(b), I show a preliminary analysis of the seismicity in Southern California over a 20 -year period. The data show a clear tendency to display a weaker decay in short times after large events. I note that although some rolloff effect at low magnitudes is expected after big shocks (implying the missing of some small magnitude events), the data in Fig. 4(b) show a change of slope in a wide magnitude range that cannot be simply attributed to this artifact. The qualitative similarity between the results from the model [Fig. 4(a)] and from actual earthquakes [Fig. 4(b)] gives additional support to the proposed fragmentation mechanism.

In summary, I have considered an analogy between a forest-fire model and the stress in a single planar fault. The inclusion of a second tree species that delays propagation of fire was shown to be analogous to including the possibility of ASs in the seismic counterpart. In the modified model, EQs (or fires) appear in clusters formed by an initial event and all its ASs. The change of exponent of the GR law is a consequence of the fact that the clusters are fragmented by the existence of ASs. An approximate case that can be analyzed with the standard percolation theory gives analytical support to this scenario. Also, observable signatures of this mechanism have been pointed out, and a preliminary analysis of actual seismic data where one of these signatures shows up has been presented.

I thank Alberto Rosso and Sebastián Risau for helpful discussions. This research was financially supported by Consejo Nacional de Investigaciones Científicas y Técnicas (CONICET), Argentina. Partial support from Grant No. PIP/112-2009-0100051 (CONICET, Argentina) is also acknowledged.

[1] B. Gutenberg and C.F. Richter, Ann. Geophys. 9, 1 (1956).

[2] C. H. Scholz, The Mechanics of Earthquakes and Faulting (Cambridge University Press, Cambridge, England, 2002).

[3] P. Bak, C. Tang, and K. Wiesenfeld, Phys. Rev. Lett. 59, 381 (1987); P. Bak, How Nature Works (Oxford University, New York, 1997); H. J. Jensen, Self-Organized Criticality (Cambridge University Press, Cambridge, England, 1998).

[4] H. Kawamura, T. Hatano, N. Kato, S. Biswas, and B. K. Chakrabarti, Rev. Mod. Phys. 84, 839 (2012).

[5] R. Burridge and L. Knopoff, Bull. Seismol. Soc. Am. 57, 341 (1967).

[6] Z. Olami, H. J.S. Feder, and K. Christensen, Phys. Rev. Lett. 68, 1244 (1992).

[7] S. Hainzl, G. Zoller, and J. Kurths, J. Geophys. Res. 104, 7243 (1999); Nonlinear Proc. Geophys. 7, 21 (2000). 
[8] E. A. Jagla and A. B. Kolton, J. Geophys. Res. 115, B05312 (2010); E. A. Jagla, Phys. Rev. E 81, 046117 (2010).

[9] S. Papanikolaou, D. M. Dimiduk, W. Choi, J. P. Sethna, M.D. Uchic, C.F. Woodward, and S. Zapperi, Nature (London) 490, 517 (2012).

[10] B. Drössel and F. Schwabl, Phys. Rev. Lett. 69, 1629 (1992).

[11] S. Hergarten, Self-Organized Criticality in Earth Systems (Springer, Berlin, 2002).

[12] A. Tejedor, J. B. Gómez, and A. F. Pacheco, Phys. Rev. E 79, 046102 (2009).

[13] P. Sinha-Ray and H. J. Jensen, Phys. Rev. E 62, 3215 (2000).

[14] W. I. Newman and D. L. Turcotte, Nonlinear Proc. Geophys. 9, 453 (2002).

[15] P. Grassberger, New J. Phys. 4, 17 (2002).

[16] G. Pruessner and H. J. Jensen, Phys. Rev. E 65, 056707 (2002)
[17] F. Omori, J. Coll. Sci., Imp. Univ. Tokyo 7, 111 (1894); T. Utsu, Y. Ogata, and R. S. Matsu'ura, J. Phys. Earth 43, 1 (1995).

[18] See Supplemental Material at http://link.aps.org/ supplemental/10.1103/PhysRevLett.111.238501 for more systematic simulations of the modified DS model, results for an uncorrelated distribution of $\mathrm{A}$ and $\mathrm{B}$ trees, and details about Fig. 4(b).

[19] D. Stauffer and A. Aharony, Introduction to Percolation Theory (Taylor \& Francis, London, 1991).

[20] M. F. Gyure and B. F. Edwards, Phys. Rev. Lett. 68, 2692 (1992).

[21] T. van Stiphout, J. Zhuang, and D. Marsan, Seismicity Declustering, Community Online Resource for Statistical Seismicity Analysis (2012), 10.5078/corssa-52382934. Available at http://www.corssa.org.

[22] M. Baiesi and M. Paczuski, Phys. Rev. E 69, 066106 (2004). 